\title{
Report
}

\section{Pilot-Study Lumina Switchable Post-Op Dressing \& Postsurgical Wounds: A Non-interventional, Non-placebo-controlled, National Pilot Study}

\author{
Michael Helmut Schmitz ${ }^{1, *}$, Sven Rogmans ${ }^{2}$, Sabine Kasparek ${ }^{2}$, Nesat Mustafi ${ }^{3}$ \\ ${ }^{1}$ Research and Studies, MCS Medical Consulting, Oberahr, Germany \\ ${ }^{2}$ Clinic for Orthopaedics, Trauma and Spinal Surgery, Krankenhaus Nordwest, Frankfurt am Main, Germany \\ ${ }^{3}$ Vascular Surgery and Vascular Medicine, Krankenhaus Nordwest, Frankfurt am Main, Germany
}

\section{Email address:}

schmitzmichael@mcsoberahr.onmicrosoft.com (M. H. Schmitz)

${ }^{*}$ Corresponding author

\section{To cite this article:}

Michael Helmut Schmitz, Sven Rogmans, Sabine Kasparek, Nesat Mustafi. Pilot-Study Lumina Switchable Post-Op Dressing \& Postsurgical Wounds: A Non-interventional, Non-placebo-controlled, National Pilot Study. Journal of Surgery. Vol. 8, No. 5, 2020, pp. $153-157$.

doi: $10.11648 /$ j.js.20200805.12

Received: June 1, 2020; Accepted: August 12, 2020; Published: September 3, 2020

\begin{abstract}
Dressings applied to primarily closed surgical incisions should be appropriate interactive dressing [13] and demonstrate an ability to protect the wound from contaminants and trauma, manage exudate, and avoid excessive pressure to the incision line. Additionally, they should be flexible, well fixed to the skin on application, skin protective (e.g. reduce the risk of blistering or irritation, not excessively adhesive) and waterproof [14]. Film dressings were one of the first modern wound-dressing products. They are extremely flexible, transparent and adhesive. During the removal of the dressing skin stress occurs. An essential role are shearing and friction forces between skin and wound dressing playing. This problem includes missing elasticity, flexibility and too strong adhesion. A non-interventional, non-placebo-controlled, national pilot study was done to prove a new shearing force reducing technique and dressing. The used post-op dressing consists of a thin film backing with a switchable polyurethane adhesive and non-adhering absorption pad. Before removal the adhesion is reduced by illuminating the dressing with the UV-A lamp. 52 patients at a mean age of 55.79 years $(\mathrm{SD} \pm 16.22)$ with a total of 52 wounds were included in the study, all of them finalized the 1-week study period. At baseline, pain was measured with a mean of 3.52 ( $\mathrm{SD} \pm 1.26$ ), during the study period pain decreased to a mean of 2.10 ( $\mathrm{SD} \pm 0.97)$ (VAS $0=$ no pain, 10=excruciating pain). Significance was calculated as 0,000 (paired t-test). At each dressing change, pain level after dressing change was rated lower compared to the pain level before dressing change. Overall, pain level decreased over the study period. The removal of the dressing after illumination with the UV-A lamp was rated in mean with 1.79 (2. Visit) and 1.86 (3. Visit) on the 6-point-scale ( $1=$ very good $-6=$ insufficient). Significance was calculated as 0,000 for visit 3 (one sample t-test [test value $=3,0]$ ). Reactions on the wound edge/wound surroundings have not been reported. In contrast, a reduction of maceration and redness of the wound edge/wound surrounding was observed. The results confirm that the adhesive ensures a safe and effective occlusion/fixation of the dressing and that the strong adhesion enables dressing to function according to specification. There was no deterioration of the wound situation or an infection in any of the cases. The switchable function of the adhesive allows the adhesion of the post-op dressing to the skin to be reduced when illuminated by the supplied UV-A lamp. The results of the present study show that the reduced adhesion upon illumination enables easy and comfortable removal of the dressing.
\end{abstract}

Keywords: Postsurgical Wound Dressing, Primarily Closed Wounds, Shearing Forces, Illumination with UV-Lamp, Reduction Stress During Dressing Change 


\section{Introduction}

Optimal management of post-operative wounds in the community is important to prevent potential complications such as surgical-site infections and wound dehiscence from developing. As such, general practitioners, who play an important part in the sub-acute management of post-operative wounds, should appreciate the physiology of wound healing and the principles of post-operative wound care [1].

There are two main types of wound healing: primary healing and secondary healing. Most surgical wounds undergo primary closure in which there is minimal tissue loss and the wound edges can be satisfactorily approximated. This allows for primary healing in which there is rapid epithelialization of the wound and minimal scarring [2].

Dressings applied to primarily closed surgical incisions should demonstrate an ability to protect the wound from contaminants and trauma, manage exudate, and avoid excessive pressure to the incision line [3].

Medical adhesive-related skin injury (MARSI) is a revalent, under-recognized and preventable complication that occurs across all care settings, age groups and patient types, from healthy patients in ambulatory care, to patients with multiple comorbidities in critical care [9].

When superficial layers of skin are removed by medical adhesive, it not only affects skin integrity, but causes pain, increases risk of infection, and potentially increases wound size and delays healing, all of which reduce patient quality of life [10]. In some cases, adhesives can also cause deeper tissue injuries beyond the loss of superficial skin layers [11]. Skin stripping and skin tears were the most common MARSI, followed by tension blisters and dermatitis [12].

Film dressings were one of the first modern wound-dressing products. They are extremely flexible, transparent and adhesive. They have no capacity to absorb fluid but are able to 'breathe off' small amounts of fluid by a process known as moisture vapor transpiration (MVT) [1]. The rate of wound infections is not increased when polyurethane film surgical dressing a polyurethane film surgical dressing is used following surgery [4]. By adding an absorbing pad, fluids can be handled and thus a dressing can be used for covering post-surgical wounds.

Films with or without absorbing pads are an extremely versatile dressing type that can be effectively used in the treatment of many superficial wounds, such as skin grafts, surgical wounds and superficial burns; they provide an optimal moist environment to promote healing, act as a barrier to bacteria, and afford protection from urine and faecal contamination [5].

During the removal of the dressing skin stress occurs. An essential role are shearing and friction forces between skin and wound dressing playing [6]. This problem includes missing elasticity, flexibility and too strong adhesion $[6,7]$.

In the EWMA-Document "Pain at wound dressing changes" there was complete agreement that gauze was the product which most often caused pain at dressing changes (mean rank=1.0), followed by knitted viscose (3.1), film dressings
(3.2), paraffin tulle (3.5) and low adherent dressings (4.8). Foam dressings and hydrocolloids were ranked equally (mean rank=6.5). Hydrogels (mean rank=9.5), hydrofibre (9.2), alginates (7.3) and soft silicones (7.2) were assessed as the products least likely to cause pain at dressing changes [8].

When removing a patient's dressing, every attempt should be made to avoid unnecessary manipulation of the wound and to prevent further damage to the delicate healing structures within the wound and surrounding skin [8].

Dressings constructed with so-called switching adhesive have lately been developed. They offer two adhesive levels, one (strong) during clinical use and one (reduced) for the removal process. The switching between the two levels is done by illumination with a UV-A lamp. The switching process is done by the nurse and is completed within seconds.

The present study was initiated to evaluate if the switchable formulation of the adhesive ensures a durable and effective adhesion strength in the range of patient- and user satisfaction on patients with postsurgical wounds for routine wound care of postsurgical wounds. Illumination of the post-op dressing with the UV-A lamp irreversibly switches the adhesion from strong to weak and guarantees a painless/comfortable removal of the dressing.

\section{Methodology}

\subsection{Study Design and Population}

A non-interventional, non-placebo-controlled, national pilot study was designed. The pilot study evaluated suitability for use as well as user and patient satisfaction during use of the tested film on patients with postsurgical wounds. This is the follow-up study of the study of the Pilot-Study ,Switchable Film Dressing \& NPWT: A non-interventional, non-placebo-controlled, national pilot study" which has been given an approval of an ethical committee (Ethics committee FEKI Freiburg, feki-code 0218/1098 05. February 2018).

The sample size was aimed at 50 patients recruited from centers and was not based on a statistical consideration.

As clinical evaluations are descriptions of practice and do not have comparators or control groups, cause and effect relationships cannot be inferred. Due to the specific nature of the study extensive statistical analysis was not planned.

The used post-op dressing consists of a thin film backing with a switchable polyurethane adhesive and non-adhering absorption pad. The dressing is breathable, allowing good oxygen and moisture vapor exchange. It is shower proof and impermeable to bacteria. An intact dressing protects the site from outside contamination. The dressings were applied and removed by healthcare professionals. Before removal the adhesion is reduced by illuminating the dressing with the UV-A lamp. Dressing change frequency was depending on the patient and wound condition and was at the discretion of the clinician. For wound cleansing and dressing application the center's current protocol was used. A total of 53 patients were continuously enrolled in the study from February 2019- 
August 2019 with the following criteria:

Inclusion criteria: individuals aged $18-85$ y.o. with postsurgical wounds.

Exclusion criteria: Untreated PAOD, Sensitivity against UV-A, Allergy against film dressing or acrylate adhesive, Pregnancy or lactation and/or Uncontrolled systemic disease.

\subsection{Study Design and Treatment Protocol}

Patients who qualified for the study were selected at the study centers. After an eligibility check and signing informed consent, the evaluation and study procedures were started. The frequency of dressing changes was at the discretion of the clinician and depended on the wound condition and the amount of exudate produced. The study product was used for a period of 1 week ( \pm 2 days).

\subsection{Data Analysis}

During evaluation, the questions are assigned to the various test parameters and evaluated according to the question category.

The question categories include:

Questions that are to be answered with yes or no: If the analysis shows more than $50 \%$ positive answers for the device the tested product characteristic or claim is to be considered as passed.

Questions requiring an assessment are assigned on a point system (very $\operatorname{good}=1$, good $=2$, satisfactory $=3$, sufficient $=4$, deficient $=5$, insufficient $=6$ ): If the evaluation results in a mean value of less than 3.0, the tested product characteristic or claim is to be considered as passed.

According to the data distribution parametric or non-parametric tests were used. An analysis of variance (ANOVA) or a paired $\mathrm{T}$ test was used for comparison if meaningful. Confidence intervals were set at $95 \%$ and results were considered significant where $p \leq 0.05$. All cases that were withdrawn were regarded as unsuccessful in terms of treatment and all variables. Study data analysis was performed independently and blinded.

\section{Results}

\subsection{Demographic and Baseline Characteristics}

$\mathrm{N}=52$ patients at a mean age of 55.79 years $(\mathrm{SD} \pm 16.22)$ with a total of 52 wounds were included in the study, all of them finalized the 1 -week study period. Gender distribution was 32 males $(61.5 \%)$ and $20(38.5 \%)$ female patients.

Mean wound age was measured with 4.92 days ( $\mathrm{SD} \pm 4.28)$. The reasons of the postsurgical wounds are shown in graph 1 .

Wound size in mean was measured with $11.08 \mathrm{~cm}^{2}$ and $0.32 \mathrm{~cm}$ depth $\left(=3.55 \mathrm{~cm}^{3}\right)$. Wound volume was reduced to a mean of $2.06 \mathrm{~cm}^{3}\left(12.09 \mathrm{~cm}^{2}\right.$ size and $0.17 \mathrm{~cm}$ wound depth) at the last visit.

The patients wound characteristics are shown in Table 1.
Wound bed condition (multiple answers possible) was rated as granulation (7), sloughy (5) and necrotic tissue (1). Level of exudation was assessed to be severe in no case, moderate in 17 cases and as light in 34 cases. Wound pain/adjacent to the wound pain was specified with a mean of 3.52 on the $\operatorname{VAS}(0=$ no pain, $10=$ excruciating pain $)$.

\subsection{Results and Analysis of Primary Parameter}

The application of the dressing with the dressing was rated in mean with 1.06 (1. Visit) and 1.08 (Visit 2) on the 6point-scale $(1=$ very good $-6=$ insufficient $)$. Significance was calculated as 0,000 for visit 1 (one sample t-test [test value $=3,0]$ ).

The adhesion was rated in mean with 1.08 (1. Visit and 2. Visit) on the 6 -point-scale ( $1=$ very good $-6=$ insufficient). Significance was calculated as 0,000 for visit 1 (one sample t-test [test value $=3,0]$ ).

Table 1. Baseline characteristics.

\begin{tabular}{|c|c|c|}
\hline $\mathrm{N}=\mathbf{5 3}$ & \multicolumn{2}{|c|}{ Mean or N (\%) } \\
\hline Age: (years) & \multicolumn{2}{|c|}{$55,79(\mathrm{SD} \pm 16.218)$} \\
\hline Range, median & \multicolumn{2}{|c|}{$22-84,56,5$} \\
\hline Sex: M/F & \multicolumn{2}{|c|}{$32(61,5 \%) / 20(38,5 \%)$} \\
\hline Wound age & \multicolumn{2}{|c|}{ 4,92 days $(\mathrm{SD} \pm 4.280)$} \\
\hline \multicolumn{3}{|l|}{ Medical history } \\
\hline Condition following & Frequencies & Percentage \\
\hline Fracture of the lower leg & 3 & 5,9 \\
\hline Arthroscopy shoulder/knee & 4 & 7,8 \\
\hline Hip TEP & 3 & 5,9 \\
\hline Inguinal hernia & 3 & 5,9 \\
\hline Breast reduction & 1 & 2,0 \\
\hline Fracture heel bone/talus & 2 & 3,9 \\
\hline Fracture tibia/tibia + fibula & 2 & 3,9 \\
\hline Laparoscopy gall bladder & 1 & 2,0 \\
\hline D2-amputation & 1 & 2,0 \\
\hline Knee-TEP & 4 & 7,8 \\
\hline Stent & 1 & 2,0 \\
\hline Fracture radius & 3 & 5,9 \\
\hline Ganglion cyst & 1 & 2,0 \\
\hline Cholecystectomy & 1 & 2,0 \\
\hline Caesarean section & 1 & 2,0 \\
\hline interior meniscus & 1 & 2,0 \\
\hline Bypass lower leg & 1 & 2,0 \\
\hline Amputation unspecified & 2 & 3,9 \\
\hline Donor site & 1 & 2,0 \\
\hline Skin lesion & 1 & 2,0 \\
\hline Femoral neck/femoral fracture & 4 & 7,8 \\
\hline Dorsum of the hand & 1 & 2,0 \\
\hline Humerus fracture & 2 & 3,9 \\
\hline Laceration lower leg & 1 & 2,0 \\
\hline Open fracture lower leg & 1 & 2,0 \\
\hline Cut wound D2 & 1 & 2,0 \\
\hline Forearm fracture & 1 & 2,0 \\
\hline Unspecified & 3 & 5,9 \\
\hline Missing data & 2 & \\
\hline
\end{tabular}


Table 2. Primary parameter

\begin{tabular}{|c|c|c|c|}
\hline \multirow[b]{2}{*}{ Day of treatment/visit } & Visit 1: & Visit 2 & Visit 3 \\
\hline & $\begin{array}{l}\text { day } 0 \text { screening } \\
\text { and baseline }\end{array}$ & $\begin{array}{l}\text { Day } 3-4 \pm 2 \text { days } 1 \text { st } \\
\text { dressing change }\end{array}$ & $\begin{array}{l}7 \pm 2 \text { days } 2 \text { nd } \\
\text { dressing change }\end{array}$ \\
\hline Application of the dressing mean (SD) 6-point-scale, $1=$ very good $-6=$ insufficient & $1,06(\mathrm{SD} \pm 0.240)$ & $1,08(\mathrm{SD} \pm 0,269)$ & - \\
\hline Adhesion of the dressing mean (SD) 6-point-scale, $1=$ very good $-6=$ insufficient & $1,08(\mathrm{SD} \pm 0.274)$ & $1,08(\mathrm{SD} \pm 0,269)$ & \\
\hline
\end{tabular}

\subsection{Results and Analysis of Secondary Parameters}

Wound bed condition (multiple answers possible) was rated as granulation (7), sloughy (5) and necrotic tissue (1). Wound bed condition had improved during the short course of the study with a reduction of sloughy and necrotic tissue (see figure 1).

Level of exudation was assessed to be severe in no case, moderate in 17 cases and as light in 34 cases. At the end of the study, level of exudate was reduced ( 0 severe, 2 moderate and 42 light).

Wound pain/adjacent to the wound pain was specified with a mean of 3.52 on the $\operatorname{VAS}(0=$ no pain, $10=$ excruciating pain) and was decreasing to a mean of 2.10 at the final visit (see table 2 and figure 2).

Painless/comfortable removal: At baseline, pain was measured with a mean of 3.52 ( $\mathrm{SD} \pm 1.26)$, during the study period pain decreased to a mean of $2.10(\mathrm{SD} \pm 0.97)$ (VAS $0=$ no pain, $10=$ excruciating pain). Significance was calculated as 0,000 (paired t-test). At each dressing change, pain level after dressing change was rated lower compared to the pain level before dressing change. Overall, pain level decreased over the study period.

The easiness of illuminating the dressing with the UV-A lamp (table 2) was rated in mean with 2.11 (2. Visit) and 2.18 (3. Visit) on the 6 -point-scale ( $1=$ very good $-6=$ insufficient). Significance was calculated as 0,000 for visit 2 and for visit 3 (one sample t-test [test value $=3,0]$ ).

The removal of the dressing after illumination with the UV-A lamp (table 2) was rated in mean with 1.79 (2. Visit) and 1.86 (3. Visit) on the 6-point-scale (1=very good $6=$ insufficient). Significance was calculated as 0,000 for visit 3 (one sample t-test [test value $=3,0]$ ).

The overall satisfaction with the application (table 2) was rated in mean with 1.06 (1. Visit) and 1.12 (2. Visit) on the 6point-scale $(1=$ very good $-6=$ insufficient). Significance was calculated as 0,000 for visit 1 (one sample t-test [test value $=3,0]$ ).

The overall satisfaction with the product was rated in mean with 1.10 on the 6 -point-scale ( $1=$ very good $-6=$ insufficient).

\subsection{Tolerability Results}

Reactions on the wound edge/wound surroundings have not been reported. In contrast, a reduction of maceration and redness of the wound edge/wound surrounding was observed (see table 1).

For the treatment of acute or surgical wounds and an optimal wound healing it is necessary to use a surgical wound dressing, that has a high tolerability during the wound treatment. Repeated dressing changes and removal of adhesive tapes may cause the epidermal layers to separate from each other or from the dermis. Even when dressings are removed carefully, without trauma, adhesive removal results in varying levels of superficial epidermal cells being detached, compromising skin barrier function [15]. Any reaction to wound care dressings or therapies may have an impact on patient safety, and delay or prevent wound healing. By using a product with a good tolerability, problems are minimized, and healing is not delayed [15].

\subsection{Safety Evaluation}

There were no serious adverse events (SAE) ore adverse advents (AE) observed. That accounts for a safe use in daily practice.

\section{Discussion}

Films are an extremely versatile dressing type that can be effectively used in the treatment of many superficial wounds, such as skin grafts, surgical wounds and superficial burns; they provide an optimal moist environment to promote healing, act as a barrier to bacteria, and afford protection from urine and fecal contamination [6]. During the removal of the dressing skin stress occurs. An essential role are shearing and friction forces between skin and wound dressing playing [6]. This problem includes missing elasticity, flexibility and to strong adhesion $[6,7]$. When removing a patient's dressing, every attempt should be made to avoid unnecessary manipulation of the wound and to prevent further damage to the delicate healing structures within the wound and surrounding skin [8].

The present study was initiated to evaluate if the switchable formulation of the adhesive ensures a durable and effective occlusion of the dressing, but also that the defined adhesion is in the range of patient- and user satisfaction.

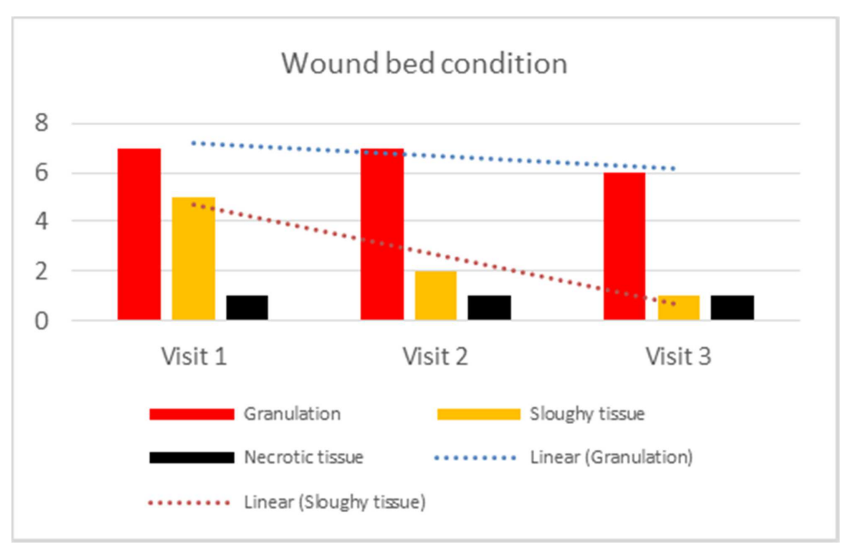

Figure 1. Evaluation of wound bed condition during the study. 


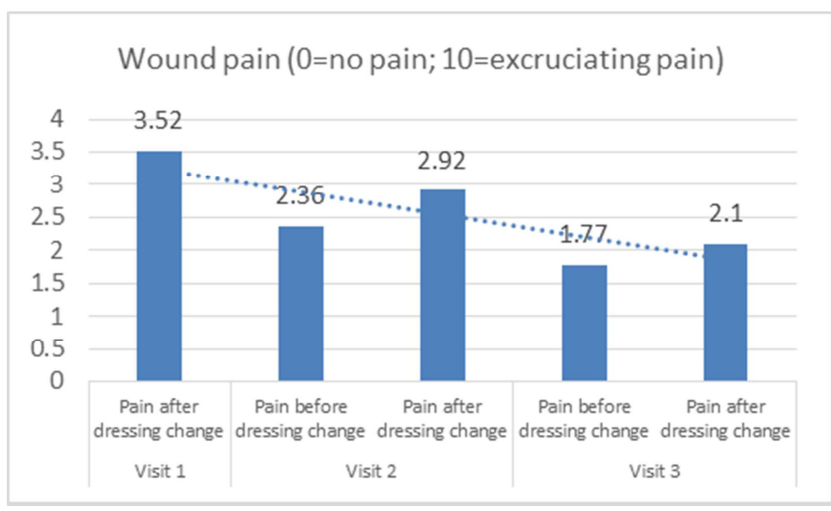

Figure 2. Evaluation of wound pain during the study.

Illumination of the post-op dressing with the UV-A lamp irreversibly switches the adhesion from strong to weak and guarantees a painless/comfortable removal of the dressing.

The number of patients and the types of wounds treated with the post-op dressing during the 1-week study period is a representative sample that provided meaningful clinical data on clinical performance of the tested post-op dressing.

The clinicians gave favorable scores for the tested post-op dressing regarding application of the dressing, overall satisfaction with the application, tightness of the dressing since the last dressing change, easiness of illuminating the dressing with the UV-A lamp and removal of the dressing after illumination with the UV-A lamp.

The overall satisfaction with the product was rated very good.

Tolerability such as pain during wearing the dressing and upon removal were given good scores. Existing wound edge and wound surroundings findings like maceration, redness etc. at visit 1 decreased. Skin irritation caused in the use of the post-op dressing did not occur.

\section{Conclusion}

The results confirm that the adhesive ensures a safe and effective occlusion/fixation of the dressing and that the strong adhesion enables dressing to function according to specification.

There was no deterioration of the wound situation or an infection in any of the cases.

The switchable function of the adhesive allows the adhesion of the post-op dressing to the skin to be reduced when illuminated by the supplied UV-A lamp. The results of the present study show that the reduced adhesion upon illumination enables easy and comfortable removal of the dressing. In sum, the use of the post-op dressing promises a a safe and tolerable use with benefits for the user and an increase of patient's quality of life.

\section{Acknowledgements}

I would like to give to $\mathrm{N}$. Mustafi my sincerest gratitude for the patient guidance and his experience in the conduct of this research.

\section{References}

[1] Kaihan Yao. Post-operative wound management. AUSTRALIAN FAMILY PHYSICIAN VOL. 42, NO. 12, DECEMBER 2013.

[2] Velnar T, Bailey T, Smrkolj V. The wound healing process: an overview of the cellular and molecular mechanisms. J Int Med Res 2009; 37: 1528-42.

[3] Bryant RA, Nix DP. Acute \& Chronic Wounds: Current Management Concepts. 5th ed. St. Louis, MO: Elsevier; 2016.

[4] Arroyo AA, Casanova PL, Soriano JV, Torra I Bou JE. Open-label clinical trial comparing the clinical and economic effectiveness of using a polyurethane film surgical dressing with gauze surgical dressings in the care of post-operative surgical wounds. Int Wound J. 2015; 12 (3): 285-292. doi: 10.1111/iwj.12099.

[5] Meuleneire F. A vapour-permeable film dressing used on superficial wounds. Br J Nurs. 2014 Aug 12; 23 (15): S36, S38-43. doi: 10.12968/bjon.2014.23. Sup15.s36.

[6] Ousey K, Wasek S Clinician perspectives on medical adhesive-related skin injuries. Wounds UK Vol 12 No 42016.

[7] Davies P, Rippon M: Evidence review: the clinical benefits of safetac technology in wound care. J Wound Care 2008; Suppl: 3-31 MEDLINE.

[8] EWMA Position Document: Pain at wound dressing changes. ${ }^{\circledR}$ MEDICAL EDUCATION PARTNERSHIP LTD.

[9] McNichol L, Lund C, Rosen T, Gray M (2013). Medical Adhesives and Patient Safety: State of the Science: Consensus statements for the assessment, prevention, and treatment of adhesive-related skin injuries. J Wound Ostomy Continence Nurs 40 (4): 365-80.

[10] Cutting KF (2008). Impact of adhesive surgical tape and wound dressings on the skin, with reference to skin stripping. J Wound Care 17 (4): 157-8, 160-2.

[11] Denyer J (2011). Reducing pain during the removal of adhesive and adherent products. Br J Nurs 20 (suppl 15): S28-S35.

[12] Maene B (2013). Hidden costs of medical tape induced skin injuries. Wounds UK 9 (1): 46-50.

[13] National Institute for Health and Clinical Excellence (NICE). Surgical site infection: evidence update April 2019. www.nice.org.uk/guidance/ng125/resources/surgical-site-infec tions-prevention-and-treatment-pdf-66141660564421. Accessed August 11, 2020.

[14] Incision care and dressing selection in surgical wounds: Findings from an international meeting of surgeons. Wounds $\begin{array}{llll}\text { International } & 2019 & \text { July } & 2019\end{array}$ www.woundsinternational.com/resources/details/incision-care -and-dressing-selection-surgical-wounds-findings-internationa 1-meeting-surgeons. Accessed August 11, 2020.

[15] Ewart J: How to identify and treat allergies to wound therapies. Wound Essentials 2015, Vol 10 No 2. 